\title{
Impaired mRNA Expression of the Migration Related Chemokine Receptor CXCR4 in Mesenchymal Stem Cells of COPD Patients
}

\author{
K. Karagiannis, ${ }^{1,2}$ A. Proklou, ${ }^{1,2}$ E. Tsitoura, ${ }^{1}$ I. Lasithiotaki, ${ }^{1}$ C. Kalpadaki, ${ }^{3}$ \\ D. Moraitaki, ${ }^{2}$ I. Sperelakis, ${ }^{4}$ G. Kontakis, ${ }^{4}$ K. M. Antoniou, ${ }^{1,2}$ and N. Tzanakis ${ }^{2}$ \\ ${ }^{1}$ School of Medicine, Laboratory of Molecular and Cellular Pneumonology, University of Crete, Heraklion, Crete, Greece \\ ${ }^{2}$ Department of Respiratory Medicine, University Hospital of Heraklion, Heraklion, Crete, Greece \\ ${ }^{3}$ Department of Haematology, University Hospital of Heraklion, Heraklion, Crete, Greece \\ ${ }^{4}$ Department of Orthopedics, University Hospital of Heraklion, Heraklion, Crete, Greece
}

Correspondence should be addressed to K. Karagiannis; k.karagiannis@med.uoc.gr

Received 31 January 2017; Accepted 6 June 2017; Published 19 July 2017

Academic Editor: Sarah Howie

Copyright $\odot 2017$ K. Karagiannis et al. This is an open access article distributed under the Creative Commons Attribution License, which permits unrestricted use, distribution, and reproduction in any medium, provided the original work is properly cited.

Defective tissue repair and remodeling are main aspects of Chronic Obstructive Pulmonary Disease (COPD) pathophysiology. Bone marrow mesenchymal stem cells (BM-MSCs) have been implicated in this direction, as their functional impairment and recruitment could possibly contribute to disease development and progression. The present study characterizes for the first time the expression of migration related chemokine receptors and their ligands in BM-MSCs from COPD patients. CXCR4/SDFla and CCR7/CCL19-CCL21 mRNA levels were evaluated in BM-MSCs obtained from twelve COPD patients and seven healthy donors. SDFla protein levels in sera and BM-MSCs' conditioned media were also evaluated. CXCR4, SDFla, CCL19, and CCL21 mRNA levels were significantly reduced in COPD BM-MSCs while CCR7 levels were undetectable. Notably, SDFla protein levels were marginally elevated in both patient sera and BM-MSCs' conditioned media while the increase in SDFla serum levels significantly correlated with disease severity in COPD. Our findings show posttranscriptional regulation of SDFla levels in BM-MSCs of COPD patients and significant downregulation of SDFla and CXCR4 mRNA indicating an involvement of the SDFla signaling pathway in the disease pathophysiology.

\section{Background}

Chronic Obstructive Pulmonary Disease (COPD) is defined as a "common preventable and treatable disease characterized by persistent airflow limitation that is usually progressive and associated with an enhanced chronic inflammatory response in the airways and the lung to noxious particles or gases." Exacerbations and comorbidities contribute to the overall severity in individual patients [1]. COPD is one of the leading causes of death globally, with huge social and economic burden [2]. Thus, it is estimated to be the fifth most common cause of morbidity and the third leading cause of death worldwide by 2030 [3]. Tobacco smoking, being well established as the main etiological factor for the development of COPD, induces a vicious circle of epithelial damage, impaired tissue repair, and remodeling. However, despite the plethora of research studies in COPD, the exact pathophysiological mechanisms are not yet fully understood.

Mesenchymal stem (stromal) cells (MSCs) are multipotent stromal cells that can be isolated from bone marrow, skeletal muscle, amniotic fluid, and adipose tissue, among others tissues. They are plastic adherent cells with a differentiation potential towards adipocytes, chondrocytes, and osteoblasts [4]. Waterman et al. have recently proposed that MSCs may be polarized into a proinflammatory MSC1 population or immunosuppressive MSC2 phenotype, dependent on their exposure to specific stimuli [5]. Therefore, it is likely that MSCs play a crucial role in both the maintenance of 
epithelial integrity and the pathogenesis of lung diseases. During the last few years a number of experimental data on animal models have showed MSCs' contribution to tissue regeneration in certain lung diseases such as elastase-induced emphysema, homing to sites of asbestos-induced lung injury, tissue remodeling in pulmonary hypertension, decrease in chronic airway inflammation in asthma, and restoration of alveolar and lung fluid balance after endotoxin-induced acute lung injury [6-11]. Consequently, great interest in the therapeutic potential of ex vivo expanded MSC has been noticed for the treatment of many disease states [12-14]. Bone marrow-derived MSCs are activated and migrate to sites of injury via mechanisms that have not yet been fully elucidated. The proposed mechanism of attraction at the sites of lung injury is via the production of soluble factors such as granulocyte colony-stimulating factor and granulocytemacrophage colony-stimulating factor, which lead to MSC proliferation and migration via the stromal cell derived factor- (SDF-) 1-CXCR4 axis [15, 16]. Similarly, previous data from Antoniou et al. showed overexpression of CXCR4 in patients with Idiopathic Pulmonary Fibrosis (IPF) [17]. Additionally, CCR7 and its ligands CCL19 and CCL21 have recently been suggested as another chemotactic axis for MSCs [18, 19]. Hence, changes in chemokines and their receptors expression by stem cells can represent defects on the migratory ability of those cells.

The aim of the current study is to investigate the possible involvement of BM-MSCs in the pathophysiology of COPD by evaluating the expression of genes involved in proliferation/migration process, namely, the CXCL12a/CXCR4 and CCR7/CCL19, CCL21 ligand/receptor dyad.

\section{Methods}

2.1. Ethics and Patient Data. This study was conducted in accordance with the amended Declaration of Helsinki. The Research Ethics Committee of the University of Crete approved the study protocol and written informed consent was obtained from the 19 subjects before entering the study (Decision 412, 18/06/2014 protocol number 14724). Of these, the 12 participants diagnosed as having COPD, according to the GOLD guidelines, were current or ex-smokers who had at least a 20-pack-year smoking history and had an FEV1/FVC ratio of $<70 \%$ and reversibility to an inhaled beta2 agonist of $<10 \%$ or $<200 \mathrm{mls}$ absolute improvement [20]. Six healthy smoking individuals, defined as smokers without airflow limitation, and one healthy ex-smoker, with an FEV1 predicted above $80 \%$, were recruited and matched for age and for smoking history, as closely as possible.

2.2. Lung Function Tests. Lung function testing, including spirometry with a bronchodilation test and DLCO measurement, was performed by all participants with a computerized system (MasterLab; 2.12, Jaeger, Wuerzburg, Germany) according to standardized guidelines.

2.3. Blood Samples Collection. Peripheral blood samples and serum were obtained from all participants and were stored at $-80^{\circ} \mathrm{C}$, after appropriate preparation, according to standard procedures.

2.4. BM-MSCs Collection and Culture. BM-MSCs were obtained from posterior iliac crest aspirates and cultured in vitro as previously described $[17,21]$. In brief, BM mononuclear cells (BMMCs) isolated with Histopaque-1077 (Sigma, Saint Louis MO) were cultured in Dulbecco's Modified Eagle Medium-Low Glucose (DMEM-LG; Gibco Invitrogen, Paisley Scotland)/10\% fetal calf serum (FCS; Hyclone, Logan, Utah, USA)/100 IU/ml penicillin-streptomycin (PS, Gibco) (MSC medium) at a concentration of $2 \times 10^{5}$ cells $/ \mathrm{cm}^{2}$ in $25 \mathrm{~cm}^{2}$ culture flasks in $37^{\circ} \mathrm{C}$ temperature $/ 5 \% \mathrm{CO} 2$ humidified atmosphere. One to three days after seeding, floating cells were removed and the medium was replaced by fresh MSC medium. Cells were passaged when $70-90 \%$ confluence was reached, using $0.25 \%$ trypsin- $1 \mathrm{mM}$ EDTA (Gibco). MSCs were identified by their morphologic and immunophenotypic characteristics and their potential to differentiate towards three different pathways, namely, adipocytes, osteocytes, and chondrocytes.

2.5. Immunophenotypic Characteristics of MSCs. BM-MSCs from second passage (P2) were immunophenotypically characterized by flow cytometry using anti-human monoclonal antibodies against anti-CD73 (AD2; Becton Dickinson-Pharmingen, San Diego, CA), anti-CDw90 (F15.42; Immunotech/Coulter), anti-CD105 (SN6; Caltag, Burlingame, CA), anti-CD45 (IMMU19.2; Immunotech/Coulter), anti-CD14 (IM2580U; Beckman-Coulter), and antiCD34 (QBend10; Beckman-Coulter). Data were processed in an Epics Elite flow cytometer (Coulter, Miami, FL).

2.6. Real-Time Reverse Transcriptase-Polymerase Chain Reaction Assay. BM-MSCs at P2 were homogenized in TRIzol reagent (Invitrogen, Carlsbad, CA), total RNA was extracted, and cDNA was synthesized by reverse transcription (RT) with the Thermoscript RT kit (Invitrogen). mRNA expression was measured using a real-time RT-PCR assay with SYBR-Green I. Glyceraldehyde-3-phosphate dehydrogenase (GAPDH) was used as the internal control, in order to normalize SDF-1a, CXCR4, CCR7, CCL19, and CCL21 expression levels. Relative expression levels per sample were calculated as $2^{-(\mathrm{Ct} \text { of gene of interest-Ct of GAPDH })}$. The mRNA-specific primers used are listed in Table 1.

2.7. Chemokine Protein Levels in Serum and BM-MSCs Conditioned Media. SDF-la levels in MSC conditioned media at P2 and sera were evaluated by means of ELISA (Quantikine; R\&D Systems, Minn., MN).

2.8. Statistical Analysis. Results were analyzed using IBM SPSS statistics v21.0. Nonparametric Mann-Whitney statistical test was used and values were expressed as medians (interquartile range). Spearman's rank coefficient (rho) was used to evaluate correlations between severity of the disease (FEV1\% pred) and chemokines expression. A value of $p<$ 0.05 was considered as statistically significant. 
TABLE 1: Primer sequences used for real-time RT-PCR.

\begin{tabular}{llcr}
\hline Gene & & Primer sequence $\left(5^{\prime}-3^{\prime}\right)$ & Annealing temperature \\
\hline \multirow{2}{*}{ CXCR4 } & FOR & GGTGGTCTATGTTGGCGTCT & $55^{\circ} \mathrm{C}$ \\
& REV & TGGAGTGTGACAGCTTGGAG & $55^{\circ} \mathrm{C}$ \\
SDFla & FOR & TGAGAGCTCGCTTTGAGTGA & $55^{\circ} \mathrm{C}$ \\
CCR7 & REV & CACCAGGACCTTCTGTGGAT & $52^{\circ} \mathrm{C}$ \\
& FOR & GGTGGTGGCTCTCCTTGTCATTTT & $57^{\circ} \mathrm{C}$ \\
CCL19 & REV & AGTTCCGCACGTCCTTCTT & $53^{\circ} \mathrm{C}$ \\
& FOR & GGACTTCCCCAGCCCCAACTCT & \\
GAPDH & FEV & TAACTGCTGCGGCGCTTCATCTT & \\
& REV & CCTCAGCTCTGGCCTCTTAC & \\
\hline
\end{tabular}

TABle 2: Patient's demographic data.

\begin{tabular}{lccc}
\hline & COPD patients $(N=12)$ & Controls $(N=7)$ & $p$ value \\
\hline Age (years) & $65.5(55.3-72)$ & $58(33-63)$ & NS \\
Gender $(\mathrm{m} / \mathrm{f})$ & $11 / 1$ & $6 / 1$ & $\mathrm{NS}$ \\
BMI & $30.1(259-31.8)$ & $27.8(25.5-31.1)$ & $\mathrm{NS}$ \\
Smoking status (c/ex) & $7 / 5$ & $30(12-80)$ & $\mathrm{NS}$ \\
PY & $53(40-78.8)$ & & \\
COPD Gold stage & & & \\
$\quad$ Stage 1 & 2 & & \\
Stage 2 & 4 & & \\
Stage 3 & 5 & & \\
Stage 4 & 1 &
\end{tabular}

Values are expressed as median (interquartile range) or frequencies; $p$ value $<0.05$ as statistically significant; $\mathrm{m} / \mathrm{f}=$ male/female; BMI $=$ Body Mass Index; c/ex $=$ current $/$ ex-smokers; $P Y=$ pack years .

\section{Results}

3.1. Demographics and Lung Function. Table 2 displays patients' data. There were no significant differences between COPD and control group in age, gender, smoking status, and history and Body Mass Index (BMI). Lung function testing results are shown in Table 3. As expected, there were statistically significant differences between COPD patients and controls in forced expiratory volume in one second (FEV1\%, 49\% versus 107\%, resp., $p<0.001$ ), forced vital capacity (FVC\%, 64\% versus 103\%, resp., $p=0.001$ ), and FEV1/FVC ratio $(60 \%$ versus $79 \%, p<0.001)$.

\subsection{BM-MSCs Identification and Immunophenotypic Charac-} teristics. BM-MSCs from both COPD and healthy subjects were cultured to P2 and subsequently were characterized by their immunophenotypic characteristics and differentiation capacity. Immunophenotypic analysis demonstrated the presence of a homogenous cell population positive for CD73, CD90, and CD105 and negative for CD45, CD14, and CD34 antigens (Table 4). Additionally, MSCs were found capable of differentiating towards the adipogenic, osteogenic, and chondrogenic lineages.
TABLE 3: Lung function test results.

\begin{tabular}{lccc}
\hline & $\begin{array}{c}\text { COPD patients } \\
(N=12)\end{array}$ & Controls $(N=7)$ & $p$ value \\
\hline FEV1\% & $49(35-72)$ & $107(80-120)$ & $<0.001$ \\
FVC\% & $64(56-81)$ & $103(87-120)$ & 0.001 \\
FEV1/FVC ratio & $60(45-70)$ & $79(75-83)$ & $<0.001$ \\
RV\% & $165(141-204)$ & $105(79-139)$ & 0.045 \\
TLC\% & $112(99-123)$ & $98(94-112)$ & NS \\
RV/TLC ratio & $56(50-69)$ & $37(28-45)$ & 0.001 \\
DLCO\% & $65(27-94)$ & $82(72-96)$ & NS \\
KCO\% & $84(43-110)$ & $90(77-106)$ & NS \\
\hline
\end{tabular}

Values are expressed as median (interquartile range); $p$ value $<0.05$ as statistically significant; FEV1 = forced expiratory volume in 1 second; FVC $=$ forced vital capacity; $\mathrm{TLCOc}=$ transfer factor of the lung for carbon monoxide; $\mathrm{KCO}=$ transfer coefficient; $\mathrm{RV}=$ residual volume; $\mathrm{TLC}=$ total lung capacity.

3.3. SDF1a/CXCR4 Axis. CXCR4 mRNA levels were reduced in BM-MSCs of COPD patients, as Figure 1(a) displays. Particularly, a significant downregulation of CXCR4 expression was detected in COPD in comparison with control group 
TABLE 4: Flow cytometry results.

\begin{tabular}{ccccccc}
\hline & CD 73 & CD 90 & CD 105 & CD 45 & CD 14 & CD 34 \\
\hline MSCs & $98.2(1.6)$ & $97.8(1.65)$ & $97.8(1.9)$ & $0.4(1.77)$ & $0.3(1.45)$ & $0(0)$ \\
\hline
\end{tabular}

Values are expressed as median (interquartile range).

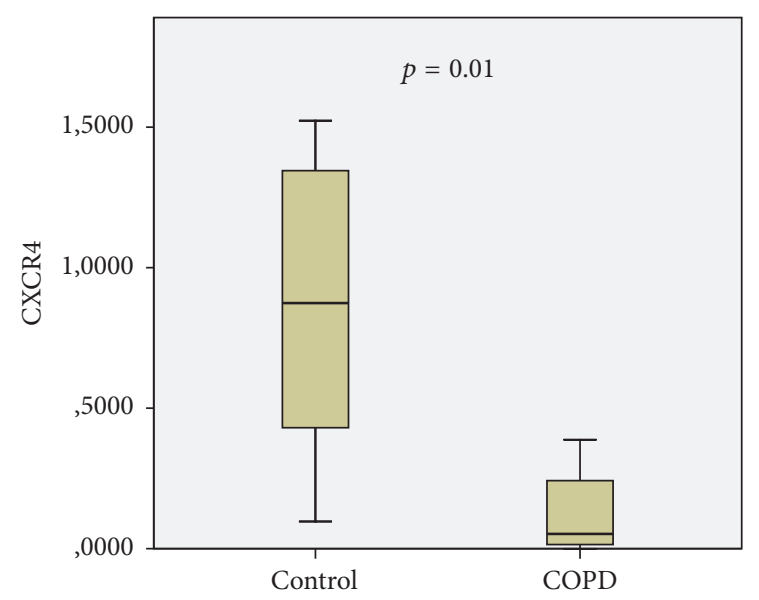

(a) CXCR4 mRNA expression by BM-MSCs

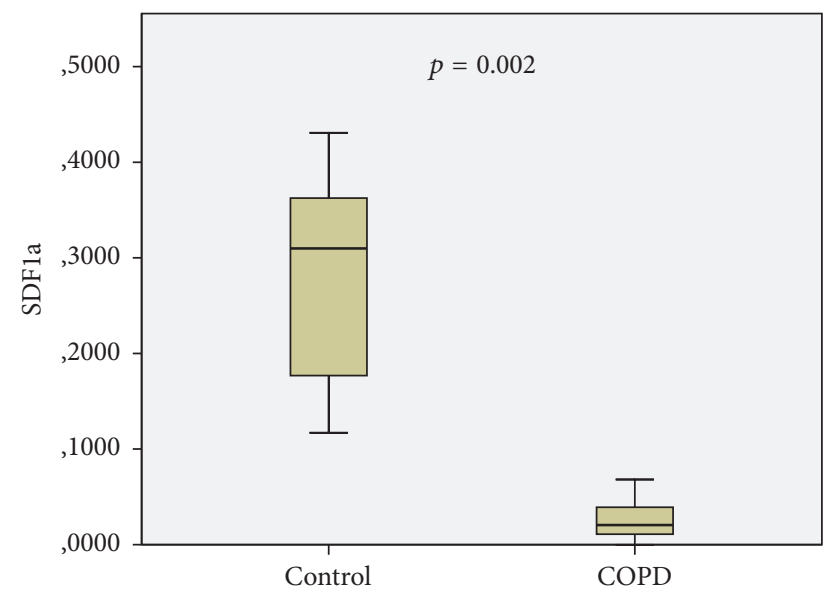

(b) SDFla mRNA expression by BM-MSCs

Figure 1

TABLE 5: Real-time RT-PCR results.

\begin{tabular}{lccc}
\hline & $\begin{array}{c}\text { COPD patients } \\
(N=12)\end{array}$ & Controls $(N=7)$ & $p$ value \\
\hline CXCR4 & $0.05(0.01-0.31)$ & $0.87(0.34-1.35)$ & 0.01 \\
SDF1a & $0.03(0.01-0.06)$ & $0.32(0.18-0.43)$ & 0.002 \\
CCL19 & $0.001(0.00-0.03)$ & $0.02(0.004-0.04)$ & 0.01 \\
CCL21 & $0.00(0.00-0.04)$ & $0.04(0.04-0.19)$ & 0.02 \\
CCR7 & No expression & No expression & NS \\
\hline
\end{tabular}

Values are expressed as median (interquartile range); $p$ value $<0.05$ as statistically significant; measurements are $2^{-\mathrm{DCT}}$; DCT $=$ Ctgene - Ctgapdh.

(0.05 versus 0.87 relative expression levels, resp., $p=0.01$, Table 5).

BM-MSCs SDFla mRNA levels were also significantly reduced in COPD relative to healthy subjects ( 0.03 versus 0.32 relative expression levels, resp., $p=0.005$, Figure $1(\mathrm{~b})$, Table 5). Interestingly, SDFla protein levels in the BM-MSCs conditioned media were found increased in the COPD group, albeit nonstatistically significant (5570 versus $2995 \mathrm{pg} / \mathrm{ml}$, $p=0.053$ ).

Additionally, serum SDFla levels were higher in COPD patients as compared to healthy subjects; however, our results did not reach statistical significance (6358 versus $3941 \mathrm{pg} / \mathrm{ml}$, $p=0.079$, Figure 2).

Notably, both SDFla mRNA expression by BM-MSCs and serum protein levels were positively correlated to the severity of disease (GOLD stages) ( $\mathrm{rho}=0.59, p=0.04$ and rho $=$ $0.63, p=0.03$, resp.). Expectedly, SDFla mRNA levels were inversely correlated to FEV1\% predicted of COPD patients (rho $=-0.57, p=0.05$, Figure 3 ).

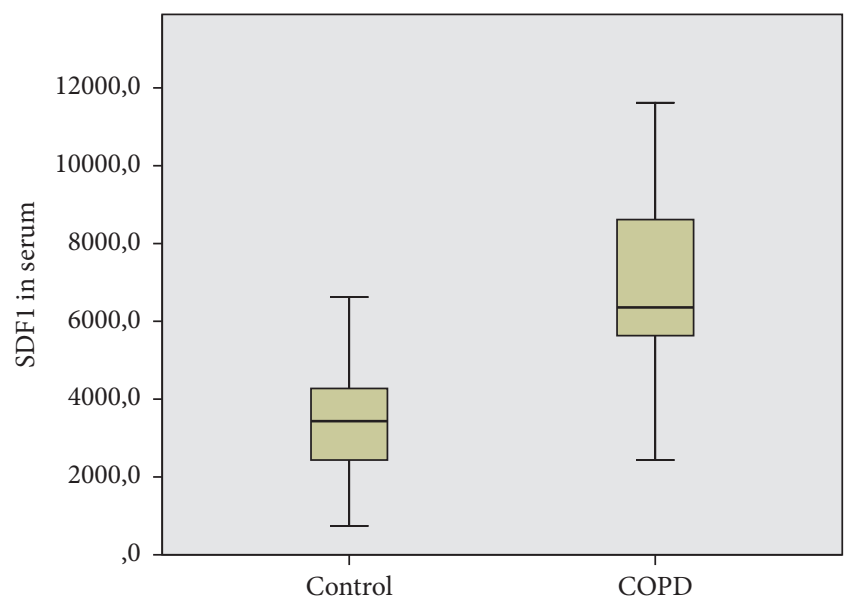

FIGURE 2: SDFla protein levels in serum.

3.4. CCR7/CCL19-CCL21 Axis. Real-time RT-PCR showed no detectable CCR7 mRNA expression in both groups. Nevertheless, its ligands' expression was downregulated in BMMSCs of COPD patients. Specifically, CCL19 (0.001 versus $0.02, p=0.01$, Figure $4(\mathrm{a})$ ) and CCL21 (0.00 versus $0.04, p=$ 0.02 , Figure $4(\mathrm{~b}))$ mRNA levels were significantly reduced in COPD BM-MSCs compared to controls (Table 5).

\section{Discussion}

Our study aims to characterize the expression of chemokines and chemokine receptors involved in the migration of mesenchymal stem cells such as the SDF1/CXCR4 and CCR7/CCL19 CCL21 axes, in BM-MSCs of COPD patients in 


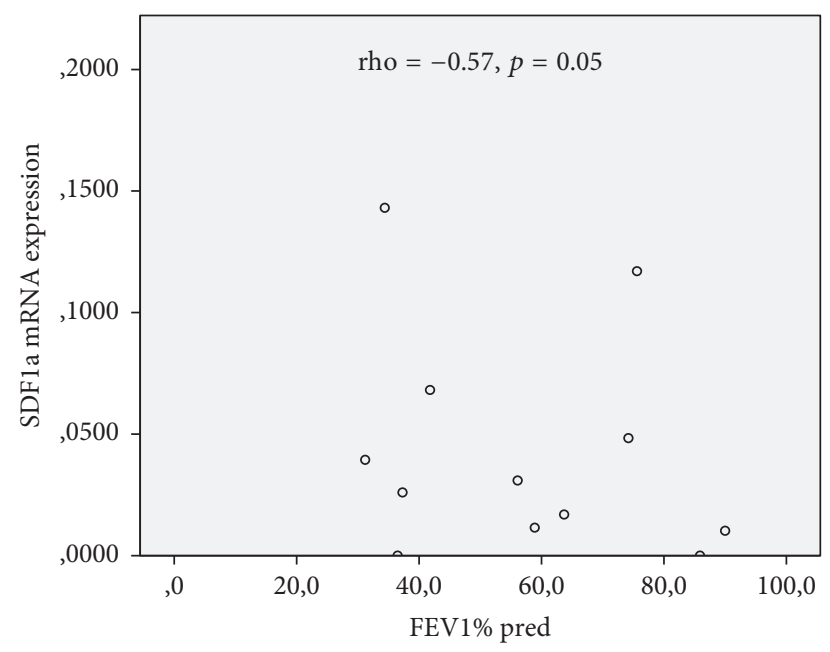

FIGURE 3: Correlations between SDFla levels and severity of disease.

comparison to healthy subjects with similar age and smoking histories. Interestingly, our results showed decreased CXCR4 mRNA expression by BM-MSCs obtained from COPD patients suggesting a possible impairment of their migratory capacity. Similarly, mesenchymal cells from the COPD group expressed lower mRNA levels of SDFla, CCL19, and CCL21 compared to controls; however, within the patient group SDFla mRNA positively correlated with disease severity. Additionally, SDFla serum protein levels were found elevated in the patient group and positively correlated to the severity of disease (GOLD stages) suggesting activation of SDFla pathway in COPD. Our study suggests therefore a role of CXCR4/SDFla axis in COPD pathophysiology that may lead to deregulation of the migration ability of stem cells from bone marrow.

Chronic inflammation and defects in repair mechanisms are considered key mechanisms in COPD development and progression of $[22,23]$. Recently, mesenchymal stem cells have been used in several animal and human studies as a potential therapeutic option for the disease, due to their anti-inflammatory and repair effect [24-27]. Our team has previously investigated the role of SDF1/CXCR4 axis in migration of BM-MSCs in Idiopathic Pulmonary Fibrosis and Rheumatoid Arthritis with Usual Interstitial Pneumonia [17, 28]. Adachi et al. showed that bone marrow transplantation had curative effect in mice with emphysema while transplantation from emphysematous mice induced the disease in normal ones, indicating a major role of bone marrow in the pathophysiology of this disease [29].

Stem cell exhaustion and senescence are considered potential pathogenetic mechanisms in COPD [30]. It could be hypothesized that an impairment of the migratory capacity of BM-MSCs could have a proinflammatory effect in COPD patients. Our results show a reduced expression of CXCR4 mRNA in BM-MSCs of COPD patients compared to healthy subjects. However, we did not find an inverse correlation between CXCR4 mRNA expression and COPD stage. Several researchers have demonstrated the importance of CXCR4 expression by mesenchymal stem cells for the mediation of their trafficking [31-33]. Recently, Yang et al. have demonstrated that CXCR4 overexpression by MSCs improves homing and colonization of damaged lung tissue and additionally suppresses the acute lung injury in animal models [31]. In another study, Bustos et al. showed that aged BM-MSCs have reduced migratory and anti-inflammatory capacity and CXCR4 is one of the genes that are downregulated in these cells [32]. The reduced mRNA expression of CXCR4 in BM-MSCs of COPD patients in our study may therefore provide evidence of a defect in migratory properties of these cells and therefore a reduced ability to mobilize and play their protective role. Liu and Xie have demonstrated that endothelial progenitor cells from COPD patients decrease in numbers and show impaired migration ability, specifically lower levels of CXCR4 expression [34]. To the best of our knowledge, the present study is the first study providing evidence that COPD affects CXCR4 mRNA expression of BM-MSCs.

Our results also showed an increase in SDFla serum levels of COPD patients, although statistical significance was not reached. Activation of the SDF1a pathway could be expected due to inflammation and tissue damage in the lung of COPD patients and our finding of elevated SDFla levels could point to this direction; however this hypothesis requires further investigation. This assumption, further supported by the positive correlation of SDFla serum levels with COPD stages, indicates an association of protein levels to the severity of the disease. SDF1 secretion from sites of tissue injury results in the increased migratory rates of progenitor cells and BMMSCs [18, 35, 36]. Zhang et al. have demonstrated that SDF1 and Hypoxia Inducible Factor-la (HIF-la) are involved in regulation of MSCs function and homing to the lungs of COPD rat models [37]. Our study demonstrates that SDF1a is also induced in COPD in humans.

Findings for SDFla expression by cultured BM-MSCs are intriguing. SDFla mRNA expression by COPD mesenchymal cells was significantly reduced compared to controls but protein levels in conditioned media were higher in COPD group. Our data imply the presence of a posttranscriptional regulation of SDFla production by BM-MSCs in COPD. It is known that MSCs are expressing SDF1 but its role has not yet been fully elucidated [38]. Lisignoli et al. have demonstrated an inverse correlation between CXCR4 and SDF1 expression by MSCs, suggesting a suppressive autocrine loop for CXCR4 expression [39]. A similar finding was reported by Pelekanos et al. in their study of fetal MSCs, but autocrine loop for CXCR4 expression was not adequate to fully explain their results [40]. It seems that there are multiple molecular pathways implicated in SDFla expression and secretion by MSCs. Interestingly, SDFla mRNA expression within the COPD group showed a positive correlation with severity of the disease similarly to the serum levels of SDFla. This result is confirmed, as expected, by a significant inverse correlation of SDFla mRNA levels with FEV1\% predicted. Both mRNA expression and secretion of SDFla by MSCs are affected in COPD patients, according to the aforementioned data; it appears though that further characterization of the regulation of SDFla expression is required. 


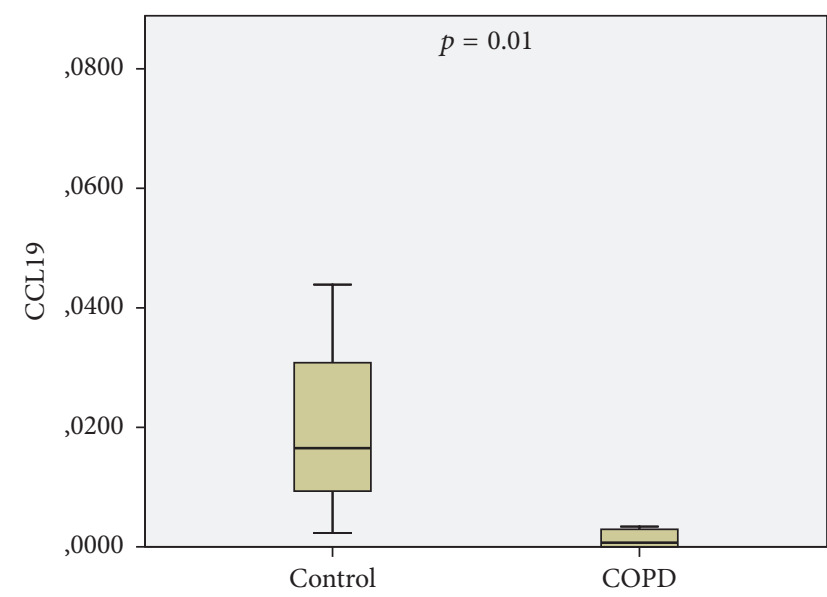

(a) CCL19 mRNA expression by BM-MSCs

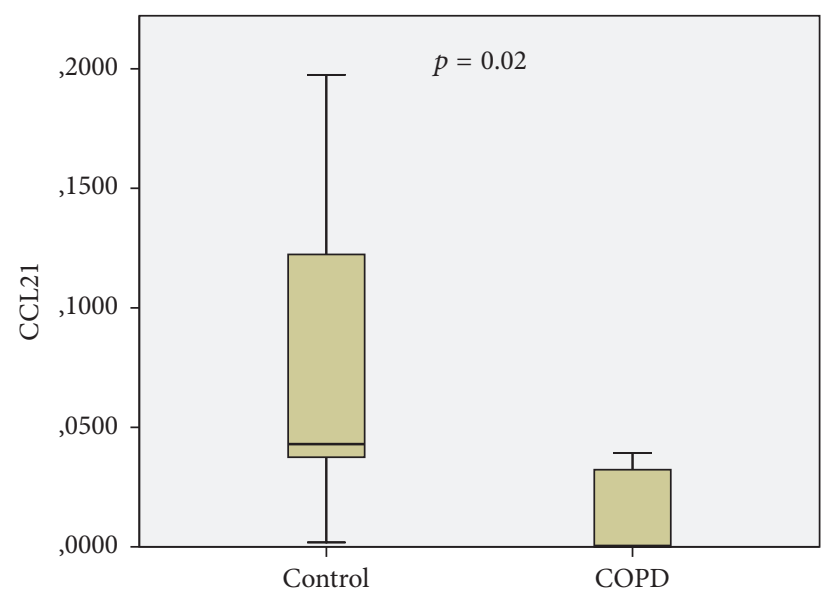

(b) CCL21 mRNA expression by BM-MSCs

Figure 4

Another chemotactic axis that has been studied as a possible regulator of mesenchymal stem cell migration is CCR7 and its ligands CCL19 and CCL21 [41]. Our results showed downregulation of both CCL19 and CCL21 mRNA transcripts in COPD patients similarly to SDFla, whereas there was no detectable expression of CCR7 in both COPD and control BM-MSCs. Although not extensively investigated, CCR7/CCL19/CCL21 axis has been also implicated in MSCs chemotaxis and tissue repair [18]. Demoor et al. have demonstrated that CCR7 plays a major role in modulating inflammatory responses in airways in COPD, and cigarette smoking upregulated CCR7, CCL19, and CCL21 mRNA expression in lymph nodes of wild-type mice [42]. CCL19 and CCL21 mRNA levels were found significantly lower in COPD BM-MSCs, similarly to the CXCR4 and SDFla mRNAs. This reduction in mRNA levels may also be associated with the senescence of BM-MSCs previously suggested as a pathogenic mechanism in COPD [30].

There are limitations in our study, which need mentioning. First, the limited sample size due to the practical difficulties of patient recruitment is noteworthy. However, this was a pilot/guide study, investigating ex vivo BM-MSCs from COPD patients which, based on our results, should be expanded to a larger patient group. Additionally, since COPD is a heterogeneous disease, different disease stages may be reflected on BM-MSCs properties and should be taken into consideration in future studies. Considering that cigarette smoking may affect the migration of BM-MSCs even in subjects without COPD, another limitation of our study is the lack of a group of healthy nonsmokers. A future study comparing the migratory capacity of BM-MSCs between smokers and nonsmokers could provide new knowledge about the effect of cigarette smoking on stem cells.

In conclusion, our study demonstrates a downregulation of the CXCR4 mRNA expression by BM-MSCs in COPD patients, providing for the first time evidence from a human study that CXCR4/SDF1 axis is dysregulated in COPD. Additionally, it shows that COPD also affects SDFla levels in serum and BM-MSCs. Although further studies are needed, we suggest that potential defects in migratory capacity of BM-MSCs may compromise their protective role, leading to development and progression of COPD.

\section{Conflicts of Interest}

The authors declare that there are no conflicts of interest regarding the publication of this paper.

\section{Acknowledgments}

The present study protocol has been awarded a grant from Hellenic Thoracic Society in 2008.

\section{References}

[1] "Global Initiative for Chronic Obstructive Lung Disease," GOLD Update, 2015.

[2] A. D. Lopez, C. D. Mathers, M. Ezzati, D. T. Jamison, and C. J. Murray, "Global and regional burden of disease and risk factors, 2001: systematic analysis of population health data," The Lancet, vol. 367, no. 9524, pp. 1747-1757, 2006.

[3] C. D. Mathers and D. Loncar, "Projections of global mortality and burden of disease from 2002 to 2030," PLoS Medicine, vol. 3, no. 11, article e442, 2006.

[4] M. E. Bernardo, F. Locatelli, and W. E. Fibbe, "Mesenchymal stromal cells," Annals of the New York Academy of Sciences, vol. 1176, pp. 101-117, 2009.

[5] R. S. Waterman, S. L. Tomchuck, S. L. Henkle, and A. M. Betancourt, "A new mesenchymal stem cell (MSC) paradigm: polarization into a pro-inflammatory $\mathrm{MSC1}$ or an immunosuppressive MSC2 phenotype," PLoS ONE, vol. 5, no. 4, Article ID e10088, 2010.

[6] K. Ishizawa, H. Kubo, M. Yamada et al., "Bone marrow-derived cells contribute to lung regeneration after elastase-induced pulmonary emphysema," FEBS Letters, vol. 556, no. 1-3, pp. 249252, 2004.

[7] J. L. Spees, D. A. Pociask, D. E. Sullivan et al., "Engraftment of bone marrow progenitor cells in a rat model of asbestosinduced pulmonary fibrosis," American Journal of Respiratory and Critical Care Medicine, vol. 176, no. 4, pp. 385-394, 2007. 
[8] V. Sueblinvong, R. Loi, P. L. Eisenhauer et al., "Derivation of lung epithelium from human cord blood-derived mesenchymal stem cells," American Journal of Respiratory and Critical Care Medicine, vol. 177, no. 7, pp. 701-711, 2008.

[9] T. L. Bonfield, M. Koloze, D. P. Lennon, B. Zuchowski, S. E. Yang, and A. I. Caplan, "Human mesenchymal stem cells suppress chronic airway inflammation in the murine ovalbumin asthma model," The American Journal of Physiology-Lung Cellular and Molecular Physiology, vol. 299, no. 6, pp. L760L770, 2010.

[10] J. W. Lee, X. Fang, N. Gupta, V. Serikov, and M. A. Matthay, "Allogeneic human mesenchymal stem cells for treatment of E. coli endotoxin-induced acute lung injury in the ex vivo perfused human lung," Proceedings of the National Academy of Sciences of the United States of America, vol. 106, no. 38, pp. 16357-16362, 2009.

[11] N. Gupta, X. Su, B. Popov, W. L. Jae, V. Serikov, and M. A. Matthay, "Intrapulmonary delivery of bone marrow-derived mesenchymal stem cells improves survival and attenuates endotoxin-induced acute lung injury in mice," Journal of Immunology, vol. 179, no. 3, pp. 1855-1863, 2007.

[12] K. Sinclair, S. T. Yerkovich, and D. C. Chambers, "Mesenchymal stem cells and the lung," Respirology, vol. 18, no. 3, pp. 397-411, 2013.

[13] X. Liu, Q. Fang, and H. Kim, "Preclinical studies of mesenchymal stem cell (MSC) administration in chronic obstructive pulmonary disease (COPD): a systematic review and metaanalysis," PLoS ONE, vol. 11, no. 6, Article ID e0157099, 2016.

[14] Z. Jin, X. Pan, K. Zhou et al., "Biological effects and mechanisms of action of mesenchymal stem cell therapy in chronic obstructive pulmonary disease," Journal of International Medical Research, vol. 43, no. 3, pp. 303-310, 2015.

[15] M. Rojas, J. Xu, C. R. Woods et al., "Bone marrow-derived mesenchymal stem cells in repair of the injured lung," American Journal of Respiratory Cell and Molecular Biology, vol. 33, no. 2, pp. 145-152, 2005.

[16] J. Xu, A. Mora, H. Shim, A. Stecenko, K. L. Brigham, and M. Rojas, "Role of the SDF-1/CXCR4 axis in the pathogenesis of lung injury and fibrosis," The American Journal of Respiratory Cell and Molecular Biology, vol. 37, no. 3, pp. 291-299, 2007.

[17] K. M. Antoniou, H. A. Papadaki, G. Soufla et al., "Investigation of bone marrow mesenchymal stem cells (BM MSCs) involvement in idiopathic pulmonary fibrosis (IPF)," Respiratory Medicine, vol. 104, no. 10, pp. 1535-1542, 2010.

[18] A. M. Hocking, "The role of chemokines in mesenchymal stem cell homing to wounds," Advances in Wound Care, vol. 4, no. 11, pp. 623-630, 2015.

[19] S. J. Baek, S. K. Kang, and J. C. Ra, "In vitro migration capacity of human adipose tissue-derived mesenchymal stem cells reflects their expression of receptors for chemokines and growth factors," Experimental \& Molecular Medicine, vol. 43, no. 10, pp. 596-603, 2011.

[20] "Global Initiative for Chronic Obstructive Lung Disease," GOLD Report, 2012.

[21] M.-C. Kastrinaki, P. Sidiropoulos, S. Roche et al., "Functional, molecular and proteomic characterisation of bone marrow mesenchymal stem cells in rheumatoid arthritis," Annals of the Rheumatic Diseases, vol. 67, no. 6, pp. 741-749, 2008.

[22] C. Noujeim and P. Bou-Khalil, "Copd updates: what's new in pathophysiology and management?” Expert Review of Respiratory Medicine, vol. 7, no. 4, pp. 429-437, 2013.
[23] M. Tsoumakidou and N. M. Siafakas, "Novel insights into the aetiology and pathophysiology of increased airway inflammation during COPD exacerbations," Respiratory Research, vol. 22, no. 7, article 80, 2006.

[24] S. Murakami, N. Nagaya, T. Itoh et al., "Adrenomedullin regenerates alveoli and vasculature in elastase-induced pulmonary emphysema in mice," American Journal of Respiratory and Critical Care Medicine, vol. 172, no. 5, pp. 581-589, 2005.

[25] N. Shigemura, M. Okumura, S. Mizuno et al., "Lung tissue engineering technique with adipose stromal cells improves surgical outcome for pulmonary emphysema," American Journal of Respiratory and Critical Care Medicine, vol. 174, no. 11, pp. 1199$1205,2006$.

[26] K. S. Schweitzer, B. H. Johnstone, J. Garrison et al., "Adipose stem cell treatment in mice attenuates lung and systemic injury induced by cigarette smoking," American Journal of Respiratory and Critical Care Medicine, vol. 183, no. 2, pp. 215-225, 2011.

[27] D. J. Weiss, R. Casaburi, R. Flannery, M. LeRoux-Williams, and D. P. Tashkin, "A placebo-controlled, randomized trial of mesenchymal stem cells in COPD," Chest, vol. 143, no. 6, pp. 1590-1598, 2013.

[28] G. A. Margaritopoulos, K. M. Antoniou, I. Lasithiotaki, A. Proklou, G. Soufla, and N. M. Siafakas, "Expression of SDF1/CXCR4 axis in bone marrow mesenchymal stem cells derived from rheumatoid arthritis-usual interstitial pneumonia," Clinical and Experimental Rheumatology, vol. 31, no. 4, pp. 610-611, 2013.

[29] Y. Adachi, H. Oyaizu, S. Taketani et al., "Treatment and transfer of emphysema by a new bone marrow transplantation method from normal mice to Tsk mice and vice versa," Stem Cells, vol. 24, no. 9, pp. 2071-2077, 2006.

[30] N. Mercado, K. Ito, and P. J. Barnes, "Accelerated ageing of the lung in COPD: new concepts," Thorax, vol. 70, no. 5, pp. 482489, 2015.

[31] J.-X. Yang, N. Zhang, H.-W. Wang, P. Gao, Q.-P. Yang, and Q.-P. Wen, "CXCR4 overexpression in mesenchymal stem cells facilitates treatment of acute lung injury in rats," The Journal of Biological Chemistry, 2014.

[32] M. L. Bustos, L. Huleihel, M. G. Kapetanaki et al., "Aging mesenchymal stem cells fail to protect because of impaired migration and antiinflammatory response," The American Journal of Respiratory and Critical Care Medicine, vol. 189, no. 7, pp. 787-798, 2014.

[33] R. J. Miller, G. Banisadr, and B. J. Bhattacharyya, "CXCR4 signaling in the regulation of stem cell migration and development," Journal of Neuroimmunology, vol. 198, no. 1-2, pp. 31-38, 2008.

[34] X. Liu and C. Xie, "Human endothelial progenitor cells isolated from COPD patients are dysfunctional," Molecular and Cellular Biochemistry, vol. 363, no. 1-2, pp. 53-63, 2012.

[35] M. Kucia, J. Ratajczak, R. Reca, A. Janowska-Wieczorek, and M. Z. Ratajczak, "Tissue-specific muscle, neural and liver stem/progenitor cells reside in the bone marrow, respond to an SDF-1 gradient and are mobilized into peripheral blood during stress and tissue injury," Blood Cells, Molecules, and Diseases, vol. 32, no. 1, pp. 52-57, 2004.

[36] A. Kortesidis, A. Zannettino, S. Isenmann, S. Shi, T. Lapidot, and S. Gronthos, "Stromal-derived factor-1 promotes the growth, survival, and development of human bone marrow stromal stem cells," Blood, vol. 105, no. 10, pp. 3793-3801, 2005.

[37] W.-G. Zhang, L. He, X.-M. Shi et al., "Regulation of transplanted mesenchymal stem cells by the lung progenitor niche in rats with chronic obstructive pulmonary disease," Respiratory Research, vol. 15, no. 1, article 33, 2014. 
[38] N. Van Overstraeten-Schlögel, Y. Beguin, and A. Gothot, "Role of stromal-derived factor-1 in the hematopoietic-supporting activity of human mesenchymal stem cells," European Journal of Haematology, vol. 76, no. 6, pp. 488-493, 2006.

[39] G. Lisignoli, S. Cristino, A. Piacentini, C. Cavallo, A. I. Caplan, and A. Facchini, "Hyaluronan-based polymer scaffold modulates the expression of inflammatory and degradative factors in mesenchymal stem cells: involvement of Cd44 and Cd54," Journal of Cellular Physiology, vol. 207, no. 2, pp. 364-373, 2006.

[40] R. A. Pelekanos, M. J. Ting, V. S. Sardesai et al., "Intracellular trafficking and endocytosis of CXCR4 in fetal mesenchymal stem/stromal cells," BMC Cell Biology, vol. 15, article 15, 2014.

[41] M. Sasaki, R. Abe, Y. Fujita, S. Ando, D. Inokuma, and H. Shimizu, "Mesenchymal stem cells are recruited into wounded skin and contribute to wound repair by transdifferentiation into multiple skin cell type," The Journal of Immunology, vol. 180, no. 4, pp. 2581-2587, 2008.

[42] T. Demoor, K. R. Bracke, K. Y. Vermaelen, L. Dupont, G. F. Joos, and G. G. Brusselle, "CCR7 modulates pulmonary and lymph node inflammatory responses in cigarette smokeexposed mice," Journal of Immunology, vol. 183, no. 12, pp. 81868194, 2009. 


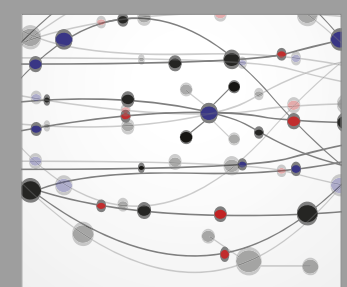

The Scientific World Journal
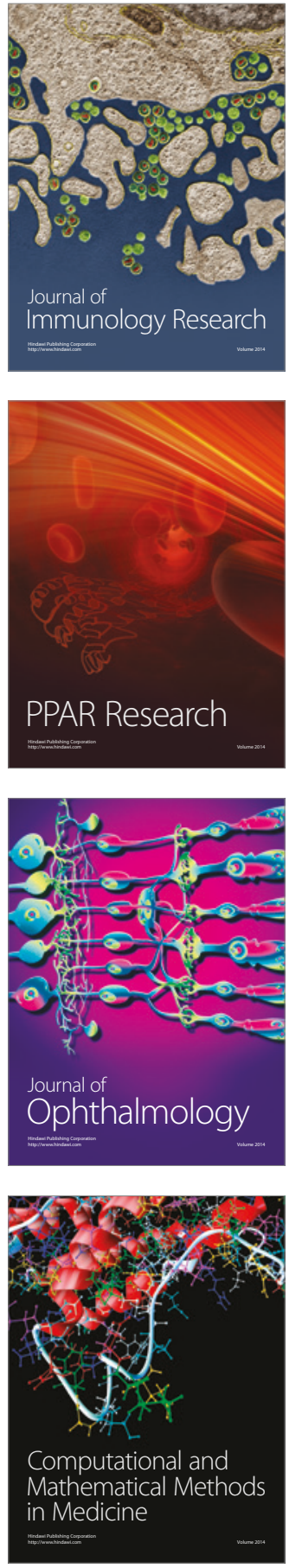

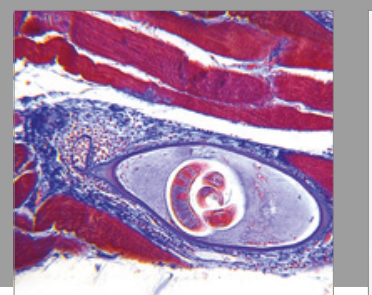

Gastroenterology Research and Practice
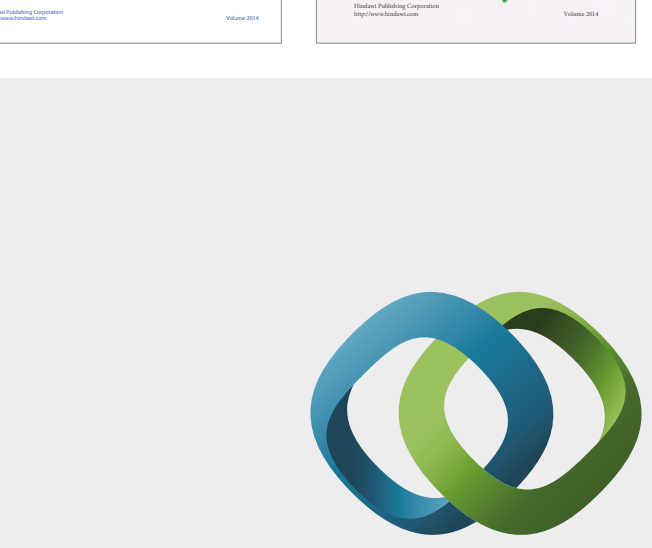

\section{Hindawi}

Submit your manuscripts at

https://www.hindawi.com
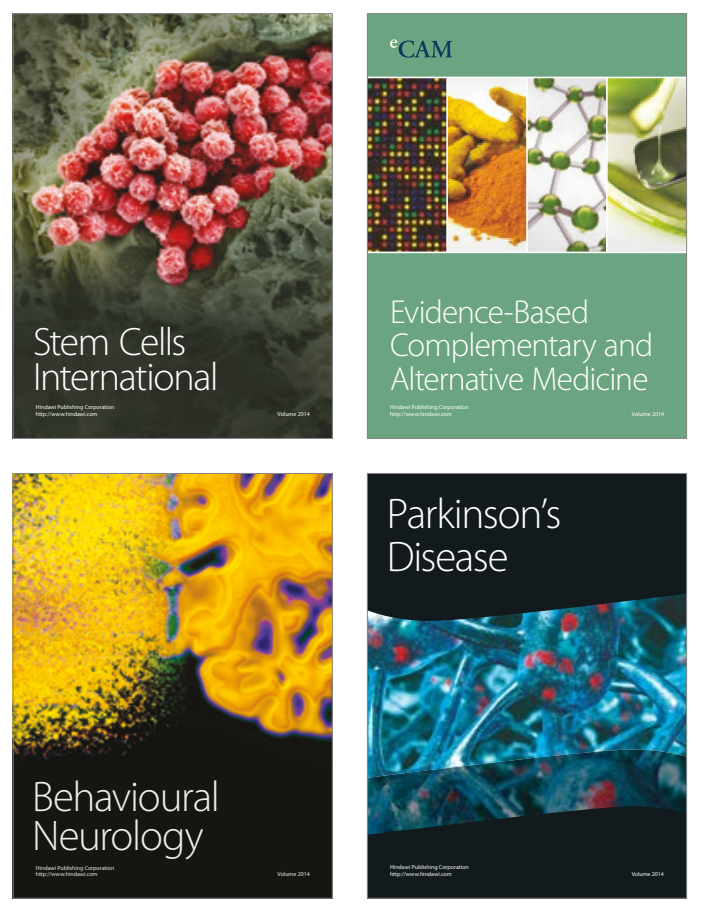
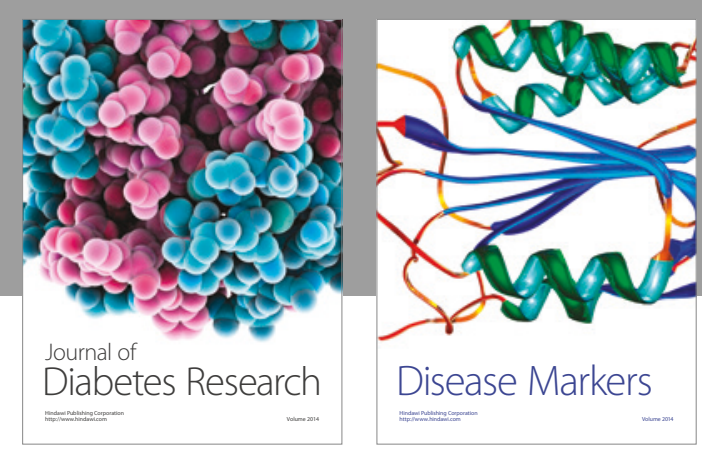

Disease Markers
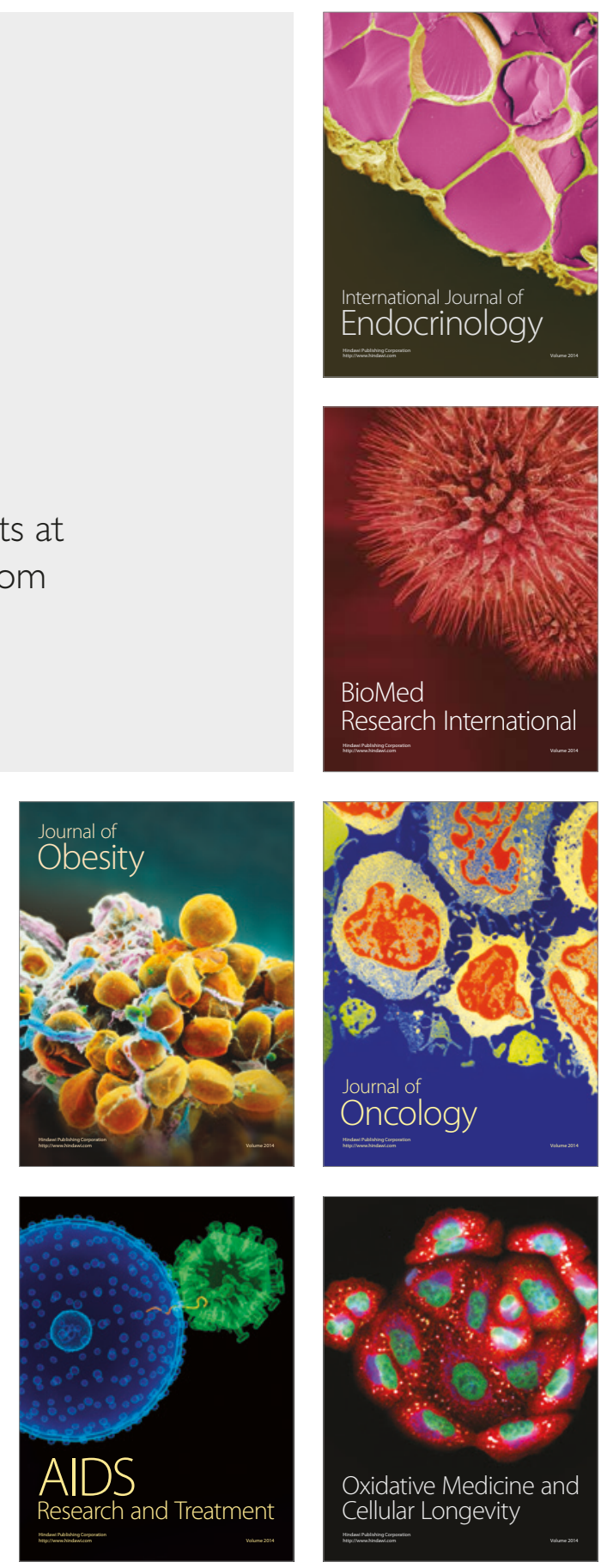